PROCEEDINGS OF THE

AMERICAN MATHEMATICAL SOCIETY

Volume 131, Number 11, Pages 3457-3461

S 0002-9939(03)06925-9

Article electronically published on February 14, 2003

\title{
A VERSION OF BURKHOLDER'S THEOREM FOR OPERATOR-WEIGHTED SPACES
}

\author{
S. PETERMICHL AND S. POTT \\ (Communicated by Joseph A. Ball)
}

\begin{abstract}
Let $W$ be an operator weight, i.e. a weight function taking values in the bounded linear operators on a Hilbert space $\mathcal{H}$. We prove that if the dyadic martingale transforms are uniformly bounded on $L_{\mathbb{R}}^{2}(W)$ for each dyadic grid in $\mathbb{R}$, then the Hilbert transform is bounded on $L_{\mathbb{R}}^{2}(W)$ as well, thus providing an analogue of Burkholder's theorem for operator-weighted $L^{2}$-spaces. We also give a short new proof of Burkholder's theorem itself. Our proof is based on the decomposition of the Hilbert transform into "dyadic shifts".
\end{abstract}

\section{INTRODUCTION}

Let $\mathcal{H}$ be a separable Hilbert space of finite or infinite dimension and let $W$ : $\mathbb{R} \rightarrow \mathcal{B}(\mathcal{H})$ be a measurable function such that $W(t)$ is positive and invertible for almost all $t$ and such that both $W$ and $W^{-1}$ are locally integrable. We call $W$ an operator weight. It gives rise to the weighted $L^{2}$ space

$$
L_{W}^{2}(\mathbb{R}, \mathcal{H})=\left\{f: \mathbb{R} \rightarrow \mathcal{H}: \int_{\mathbb{R}}\langle W(t) f(t), f(t)\rangle d t<\infty\right\} .
$$

Let $\mathcal{D}$ denote the collection of all dyadic intervals in $\mathbb{R}$. We call $\mathcal{D}$ the standard dyadic grid in $\mathbb{R}$. For each $\alpha \in \mathbb{R}, r>0$, let $\mathcal{D}^{\alpha, r}$ be the dyadic grid $\{\alpha+r I: I \in \mathcal{D}\}$. We define dyadic Martingale transforms $\left\{T_{\sigma}\right\}_{\sigma \in\{-1,1\}^{\mathcal{D}}}$ relative to the standard grid $\mathcal{D}$ by

$$
f=\sum_{I \in \mathcal{D}} f_{I} h_{I} \mapsto T_{\sigma} f=\sum_{I \in \mathcal{D}} \sigma(I) f_{I} h_{I}
$$

for functions in $L^{2}(\mathbb{R}, \mathcal{H})$ with finite Haar expansion $\sum_{I \in \mathcal{D}} f_{I} h_{I}$. Here, $\left\{h_{I}\right\}_{I \in \mathcal{D}}$ denotes the standard Haar basis and $f_{I}$ the formal Haar coefficient $f_{I}=\int_{\mathbb{R}} f h_{I} d x$. If we take a Banach space $X$ and consider dyadic martingale transforms on $L^{2}(\mathbb{R}, X)$, then uniform boundedness of the dyadic martingale transforms $\left\{T_{\sigma}\right\}_{\sigma \in\{-1,1\}^{\mathcal{D}}}$ is

Received by the editors August 19, 2001 and, in revised form, May 28, 2002.

2000 Mathematics Subject Classification. Primary 42A50, 47B37; Secondary 42A61.

Key words and phrases. Operator-weighted inequalities, Hilbert transform, martingale transforms, UMD spaces.

The second author gratefully acknowledges support by EPSRC.

(C)2003 American Mathematical Society 
equivalent to the so-called UMD-property of $X$ (i.e. $X$-valued martingale difference sequences converge unconditionally). Burkholder proved in [2] that for a Banach space $X$, the UMD property, i.e. the uniform boundedness of the dyadic martingale transforms $\left\{T_{\sigma}\right\}$ on $L^{2}(\mathbb{R}, X)$, implies the boundedness of the Hilbert transform $H$ on $L^{2}(\mathbb{R}, X)$. The same statement holds for $L^{p}(\mathbb{R}, X)$ for $1<p<\infty$. So it is natural to ask whether the uniform boundedness of martingale transforms on $L_{W}^{2}(\mathbb{R}, \mathcal{H})$ implies boundedness of the Hilbert transform. The difference to the situation of Burkholders' Theorem is that the norm on $L_{W}^{2}(\mathbb{R}, \mathcal{H})$ is no longer translation- or dilation-invariant. Already the scalar situation [4] shows that we cannot expect the uniform boundedness of the $\left\{T_{\sigma}\right\}_{\sigma \in\{-1,1\}^{\mathcal{D}}}$ to be sufficient. In the scalar situation, this is equivalent to the dyadic Muckenhoupt condition $A_{2}^{d}$, whilst boundedness of the Hilbert transform is equivalent to the Muckenhoupt condition $A_{2}$, and the two are not equivalent. This is quite natural: $A_{2}$ is the translation invariant version of $A_{2}^{d}$. It turns out that we also need dilations. We have to consider dyadic martingale transforms with respect to the dilated and translated dyadic grids $\mathcal{D}^{\alpha, r}$ for all $\alpha \in \mathbb{R}$, $r>0$. We define martingales $T_{\sigma}^{\alpha, r}$ relative to the grids $\mathcal{D}^{\alpha, r}$ as above, replacing the standard Haar system by the system $\left(h_{I^{\alpha, r}}\right)_{I \in \mathcal{D}}$. Here $h_{I^{\alpha, r}}(x)=\frac{1}{r} h_{I}\left(\frac{1}{r} x-\alpha\right)$. Here is our main result:

Theorem 1.1. Let $W$ be a $\mathcal{B}(\mathcal{H})$-valued operator weight on $\mathbb{R}$. Suppose that the martingale transforms $T_{\sigma}^{\alpha, r}$ are bounded on $L_{W}^{2}(\mathbb{R}, \mathcal{H})$ with a uniform bound for all $\sigma \in \mathcal{D}, \alpha \in \mathbb{R}$ and $r>0$. Then the Hilbert transform $H$ is also bounded on $L_{W}^{2}(\mathbb{R}, \mathcal{H})$, and there exists an absolute constant $C>0$ such that

$$
\|H\|_{L_{W}^{2}(\mathbb{R}, \mathcal{H}) \rightarrow L_{W}^{2}(\mathbb{R}, \mathcal{H})} \leq C \sup _{r, \alpha, \sigma}\left\|T_{\sigma}^{\alpha, r}\right\|_{L_{W}^{2}(\mathbb{R}, \mathcal{H}) \rightarrow L_{W}^{2}(\mathbb{R}, \mathcal{H})}^{2}
$$

Estimates of the norm of the Hilbert transform on operator weighted spaces by means of the martingale transforms are not new; see for example [6]. But the proof in [6] seems to be dimension dependent, while our method is dimension independent and very general. It relies on the decomposition of the Hilbert transform by the first author in [5].

\section{Proof of the main Result}

Proof. In [5] it is shown that the Hilbert transform $H$ on the real line $\mathbb{R}$ is contained in a multiple of the closed convex hull of the "dyadic shifts" $\left\{\amalg^{\alpha, r}\right\}_{\alpha \in \mathbb{R}, r>0}$. Here, the dyadic shift $\amalg^{\alpha, r}$ is defined by

$$
f=\sum_{I \in \mathcal{D}} f_{I^{\alpha, r}} h_{I^{\alpha, r}} \mapsto \amalg^{\alpha, r} f=\sum_{I \in \mathcal{D}} \frac{1}{\sqrt{2}} f_{I^{\alpha, r}}\left(h_{I_{+}^{\alpha, r}}-h_{I_{-}^{\alpha, r}}\right)
$$

for functions with finite Haar expansion relative to the system $\left\{h_{I^{\alpha, r}}\right\}_{I \in \mathcal{D}}$. As usual, $I_{-}$here denotes the left and $I_{+}$the right half of $I$. Notice that $\amalg^{\alpha, r}=\amalg^{\alpha, 2 r}$. So it is sufficient to show that $\left\|\amalg^{\alpha, r}\right\| \leq \sup _{\sigma}\left\|T_{\sigma}^{\alpha, r}\right\|^{2}$ for all $\alpha \in \mathbb{R}, 1 \leq r<2$. We introduce the square function $\mathcal{S}$,

$$
\mathcal{S} f(t)=\left(\int_{\Sigma}\left\|W^{1 / 2}(t)\left(T_{\sigma} f\right)(t)\right\|^{2} d \sigma\right)^{1 / 2}
$$


Here, $d \sigma$ denotes the natural product measure on $\Sigma=\{-1,1\}^{\mathcal{D}}$, which assigns equal measure $2^{-k}$ to all cylindrical sets of length $k$. But by observing that $\frac{1}{\sqrt{2}}\left(h_{I_{+}}-h_{I_{-}}\right)(t)$ differs from $h_{I}(t)$ only by a sign dependent on $I$ and $t$, it is not difficult to see that $(\mathcal{S} f)(t)=(\mathcal{S} \amalg f)(t)$, where $\amalg$ is the dyadic shift relative to the standard dyadic grid.

$$
\begin{aligned}
(\mathcal{S} \amalg f)(t)^{2} & =\int_{\Sigma}\left\|W^{1 / 2}(t) \sum_{I \in \mathcal{D}} \sigma(I) f_{I} \frac{1}{\sqrt{2}}\left(h_{I_{+}}-h_{I_{-}}\right)(t)\right\|^{2} d \sigma \\
& =\int_{\Sigma}\left\|W^{1 / 2}(t) \sum_{I \in \mathcal{D}} \sigma(I) f_{I} h_{I}(t)\right\|^{2} d \sigma \\
& =(\mathcal{S} f)(t)^{2} .
\end{aligned}
$$

Note that for any given $t$, the expression under the integral appearing in the first line of (2.1) is of the form

$$
\left\|W^{1 / 2}(t) \sum_{I \in \mathcal{D}} \sigma(I) f_{I} \frac{1}{|I|} \varepsilon_{I, t}\right\|^{2},
$$

and the expression under the next integral is of the form

$$
\left\|W^{1 / 2}(t) \sum_{I \in \mathcal{D}} \sigma(I) f_{I} \frac{1}{|I|} \tilde{\varepsilon}_{I, t}\right\|^{2},
$$

where $\varepsilon_{I, t}, \tilde{\varepsilon}_{I, t} \in\{-1,1\}$ for all $t$ and $I$. Integration over all $\sigma=(\sigma(I))_{I \in \mathcal{D}} \in \Sigma$ now gives the desired equality in (2.1). Thus we have

$$
\begin{aligned}
\|\amalg f\|_{L_{W}^{2}(\mathbb{R}, \mathcal{H})}^{2} \\
\leq \sup _{\sigma}\left\|T_{\sigma}\right\|_{L_{W}^{2}(\mathbb{R}, \mathcal{H}) \rightarrow L_{W}^{2}(\mathbb{R}, \mathcal{H})}^{2} \int_{\Sigma}\left\|T_{\sigma} \amalg f\right\|_{L_{W}^{2}(\mathbb{R}, \mathcal{H})}^{2} d \sigma \\
=\sup _{\sigma}\left\|T_{\sigma}\right\|_{L_{W}^{2}(\mathbb{R}, \mathcal{H}) \rightarrow L_{W}^{2}(\mathbb{R}, \mathcal{H})}^{2}\|\mathcal{S} \amalg f\|_{L_{W}^{2}(\mathbb{R}, \mathcal{H})}^{2} \\
=\sup _{\sigma}\left\|T_{\sigma}\right\|_{L_{W}^{2}(\mathbb{R}, \mathcal{H}) \rightarrow L_{W}^{2}(\mathbb{R}, \mathcal{H})}^{2}\|\mathcal{S} f\|_{L_{W}^{2}(\mathbb{R}, \mathcal{H})}^{2} \\
=\sup _{\sigma}\left\|T_{\sigma}\right\|_{L_{W}^{2}(\mathbb{R}, \mathcal{H}) \rightarrow L_{W}^{2}(\mathbb{R}, \mathcal{H})}^{2} \int_{\Sigma} \int_{\mathbb{R}}\left\|W^{1 / 2}(t)\left(T_{\sigma} f\right)(t)\right\|_{\mathcal{H}}^{2} d t d \sigma \\
=\sup _{\sigma}\left\|T_{\sigma}\right\|_{L_{W}^{2}(\mathbb{R}, \mathcal{H}) \rightarrow L_{W}^{2}(\mathbb{R}, \mathcal{H})}^{2} \int_{\Sigma}\left\|T_{\sigma} f\right\|_{L_{W}^{2}(\mathbb{R}, \mathcal{H})}^{2} d \sigma \\
\leq \sup _{\sigma}\left\|T_{\sigma}\right\|_{L_{W}^{2}(\mathbb{R}, \mathcal{H}) \rightarrow L_{W}^{2}(\mathbb{R}, \mathcal{H})}^{4}\|f\|_{L_{W}^{2}(\mathbb{R}, \mathcal{H})}^{2} .
\end{aligned}
$$

The first inequality here just comes from the fact that each martingale transform is inverse to itself. By the same argument with the corresponding square function, we obtain the same estimate for all dyadic grids, namely

$$
\left\|\amalg^{\alpha, r}\right\|_{L_{W}^{2}(\mathbb{R}, \mathcal{H}) \rightarrow L_{W}^{2}(\mathbb{R}, \mathcal{H})} \leq \sup _{\sigma}\left\|T_{\sigma}^{\alpha, r}\right\|_{L_{W}^{2}(\mathbb{R}, \mathcal{H}) \rightarrow L_{W}^{2}(\mathbb{R}, \mathcal{H})}^{2} .
$$

This finishes the proof of the theorem. 


\section{A short proof of Burkholder's Theorem}

The same method can be applied to prove Burkholder's Theorem.

Theorem 3.1 (Burkholder [2]). Suppose that the martingale transforms $\left\{T_{\sigma}\right\}$ are uniformly bounded on $L^{p}(\mathbb{R}, X)$ for some $1<p<\infty$. Then the Hilbert transform is also bounded on $L^{p}(\mathbb{R}, X)$ and consequently on all $L^{q}(\mathbb{R}, X)$ for $1<q<\infty$.

Proof. Because of the translation and dilation invariance of the norm on $L^{p}(\mathbb{R}, X)$, it is sufficient to show that the dyadic shift $\amalg$ is bounded on $L^{p}(\mathbb{R}, X)$. Again, introduce the Littlewood-Paley expression

$$
(\mathcal{P} f)(t)=\left(\int_{\Sigma}\left\|T_{\sigma} f(t)\right\|_{X}^{p} d \sigma\right)^{1 / p}
$$

and observe that $(\mathcal{P} \amalg f)(t)=(\mathcal{P} f)(t)$ with the same argument as above. Thus

$$
\begin{aligned}
\| & \amalg f \|_{L^{p}(\mathbb{R}, X)} \\
\leq & \sup _{\sigma}\left\|T_{\sigma}\right\|\left(\int_{\Sigma}\left\|T_{\sigma} \amalg f\right\|_{L^{p}(\mathbb{R}, X)}^{p} d \sigma\right)^{1 / p} \\
= & \sup _{\sigma}\left\|T_{\sigma}\right\|\left(\int_{\Sigma} \int_{\mathbb{R}}\left\|T_{\sigma} \amalg f(t)\right\|_{X}^{p} d t d \sigma\right)^{1 / p} \\
= & \sup _{\sigma}\left\|T_{\sigma}\right\|\|\mathcal{P} \amalg f\|_{L^{p}(\mathbb{R})} \\
= & \sup _{\sigma}\left\|T_{\sigma}\right\|\|\mathcal{P} f\|_{L^{p}(\mathbb{R})} \\
= & \sup _{\sigma}\left\|T_{\sigma}\right\|\left(\int_{\Sigma}\left\|T_{\sigma} f\right\|_{L^{p}(\mathbb{R}, X)}^{p} d \sigma\right)^{1 / p} \\
\leq & \sup _{\sigma}\left\|T_{\sigma}\right\|_{L^{p}(\mathbb{R}, X)}^{2} .
\end{aligned}
$$

It is not clear whether the reverse of this theorem, Bourgain's theorem 11, has an analogue for operator weighted $L^{2}$ spaces. The transference technique of Bourgain relies heavily on the translation invariance of $L^{2}(\mathbb{R}, X)$, which we do not have in $L_{W}^{2}(\mathbb{R}, \mathcal{H})$. A result along these lines has been obtained in [3], but it does not provide the full analogue we would wish for here.

\section{ACKNOWLEDGMENTS}

We thank A. Volberg and F. Nazarov for helpful discussions.

\section{REFERENCES}

1. J. Bourgain, Some remarks on Banach spaces in which martingale difference sequances are unconditional, Ark. Mat. 21 (1983), no. 2, 163-168. MR 85a:46011

2. D.L. Burkholder, A geometric condition that implies the existence of certain singular integrals of Banach space valued functions, Proc. Conf. Harmonic Analysis in honor of A. Zygmund, ed. W. Beckner, A.P. Calderon, R. Fefferman, P. Jones, Wadsworth, Belmont, Ca., 1983. MR 85i:42020

3. T. A. Gillespie, S. Pott, S. Treil, And A. Volberg, A transference approach to estimates of vector Hankel operators, St. Petersburg Math. J. 12 (2001), no. 6, 1013-1024. MR 2002a:47039 
4. R.A. Hunt, B. Muckenhoupt, R.L. Wheeden, Weighted norm inequalities for the conjugate function and the Hilbert transform, Trans. Am. Math. Soc. 176 (1973), 227-251. MR 47:701

5. S. Petermichl, Dyadic shifts and a logarithmic estimate for Hankel operators with matrix symbol, C. R. Acad. Sci. Paris Sér I Math. 330 (2000), 455-460. MR 2000m:42016

6. S. Treil, A. Volberg, Wavelets and the angle between past and future, J. Funct. Anal. 143 (1997), 269-308. MR 99k:42073

School of Mathematics, Institute of Advanced Studies, Einstein Drive, Princeton, NEW JERSEY 08540

E-mail address: stefanie@math.msu.edu

Department of Mathematics, University of York, York YO10 5DD, United Kingdom

E-mail address: sp23@york.ac.uk 